\title{
Inhibition of ubiquitin proteasome function prevents monocrotaline-induced pulmonary arterial remodeling
}

Yanting Zhu ${ }^{1}$, Yinxia Wu ${ }^{2}$, Wenhua Shi ${ }^{1}$, Jian Wang ${ }^{1}$, Xin Yan ${ }^{1}$, Qingting Wang ${ }^{1}$, Ya Liu ${ }^{1}$, Lan Yang ${ }^{1}$, Li Gao ${ }^{3}$, Manxiang $\mathrm{Li}^{1}$

${ }^{1}$ Department of Respiratory Medicine, the First Affiliated Hospital of Xi'an JiaoTong University, Xi'an, Shaanxi 710061, People's Republic of China

${ }^{2}$ Wuhan University of Science and Technology, Wuhan, Hubei 430081, People’s

Republic of China

${ }^{3}$ Division of Allergy and Clinical Immunology, Department of Medicine, The Johns Hopkins University School of Medicine, Baltimore MD 21224, USA

Address correspondence to:

Dr. Manxiang Li

Department of Respiratory Medicine, the First Affiliated Hospital of Xi' an Jiaotong University

No.277, Yanta West Road, Xi'an 710061, Shaanxi, PR China

Telephone: +86-029-85324053

Fax: +86-029-85324053

E-mail address: manxiangli@ hotmail.com

Word count: 5266

Figure count: 5 


\section{Abstract}

Aims: Previous study has indicated that inhibition of proteasome function ameliorates the development of pulmonary arterial hypertension (PAH), while its underlying mechanisms are still unclear. This study was performed to address these issues.

Material and methods: Male Sprague-Dawley (SD) rats were divided into five groups: control group, PAH group, vehicle treated PAH group, MG-132 treated PAH group and bortezomib treated PAH group. PAH model was established by a single intraperitoneal injection of monocrotaline (MCT). MG-132 and bortezomib were administered to inhibit proteasome function. The right ventricular systolic pressure (RVSP), the right ventricle hypertrophy index (RVHI) and the percentage of medial wall thickness (\%MT) were used to evaluate the development of PAH. Hematoxylin and eosin staining was performed to measure vascular remodeling. Immunobloting was used to determine Akt phosphorylation, expression of PTEN and NEDD4, and the level of ubiquitinated-PTEN protein.

Key findings: MCT increased RVSP, RVHI and \%MT in rats, while these changes were suppressed by treatment of PAH rats with MG-132 or bortezomib. In PAH model, expression of PTEN was decreased and phosphorylation of Akt was increased, these were accompanied by an elevation of NEDD4 protein level. Treatment of PAH model with MG-132 or bortezomib increased PTEN expression and accumulation of ubiquitinated-PTEN protein and decreased Akt phosphorylation, while didn't change NEDD4 expression.

Significance: Inhibition of proteasome function ameliorates pulmonary arterial remodeling by suppressing UPS-mediated PTEN degradation and subsequent inhibition of PI3K/Akt pathway, indicating that UPS might be a novel target for prevention of PAH.

Key words: pulmonary arterial hypertension; vascular remodeling; ubiquitin proteasome system; degradation; PTEN; NEDD4. 


\section{Introduction}

Pulmonary arterial hypertension (PAH) is a fatal disease characterized by a progressive increase in pulmonary vascular resistance, eventually resulting in right heart failure and death. Common pathological mechanisms of PAH include persistent pulmonary vasoconstriction, vascular remodeling and thrombosis in situ [1]. Vascular remodeling including intima thickening and media hyperplasia is a key pathological feature of PAH, which is majorly caused by pulmonary arterial smooth muscle cells (PASMCs) proliferation/migration, leading to narrowness and obstruction of small pulmonary arteries $[2,3]$. Therefore, it is critical to elucidate the molecular mechanisms responsible for PASMCs proliferation and to identify appropriate targets to inhibit vascular remodeling and thus to manage PAH.

Phosphatase and tensin homologue deleted on chromosome ten (PTEN), a dual lipid/protein phosphatase, regulates mammalian cellular growth [4-6]. PTEN dephosphorylates second-messenger phosphatidyl inositol-3,4,5-trisphosphate (PIP3), and subsequently inhibits class I phosphoinositide 3-kinase (PI3K)/Akt signaling mediated cellular survival and growth response $[5,7,8]$. Previous studies have shown that the functional insufficiency of PTEN due to its mutation [9] or down-regulation of its expression [10] is associated with the pathogenesis of several diseases, such as cancer, cardiac hypertrophy, diabetes and PAH [11-14]. Except genetic alterations and changes of gene transcription and translation, post-translational modifications (PTMs) also regulate particular protein functions by ubiquitination, phosphorylation, acetylation and oxidation [15-18].

Ubiquitin proteasome system (UPS), a major protein quality and quantity control system, plays a major role in non-lysosomal protein degradation [19]. The specificity of UPS is principally determined by ubiquitin ligating enzyme (E3 ligase) which is responsible for substrate recognition [20]. UPS regulates the turnover of many kinds 
of proteins which are involved in many cellular processes, including cell cycle progression, signaling transduction, gene expression and apoptosis [21-23]. Elevated function of UPS is found to be associated with the development of several types of disease, such as cancer, cardiovascular and neurological disorder [24-29]. Recent studies have indicated that UPS-mediated degradation of PTEN induces cell proliferation in prostate cancer and promotes axon branching in peripheral neurons interrupts [18, 26, 28, 29].

Proteasome inhibition is a promising therapeutic strategy for cancer treatment [30]. Additionally, several clinical trials have been running to test the effects of proteasome inhibitors on non-oncologic diseases [31]. Inhibition of proteasome function has been recently shown to inhibit PASMCs proliferation in vitro [32]. Study in animal model has also found that proteasome inhibitors are capable of reducing the thickness of pulmonary vascular walls in chronic hypoxia and chronic hypoxia/SU-5416 models of PAH in rats [33]. However, it is unclear whether regulation of PTEN mediates the effects of proteasome inhibition to benefit PAH. This study aims to examine this and to further explore its potential mechanisms.

\section{Materials and Methods}

\section{Materials}

MCT was supplied by Sigma-Aldrich (St. Louis, MO, USA). MG-132

(Cbz-Leu-Leu-Leu-aldehyde, Z-LLL-H), a selective proteasome inhibitor, was purchased from Selleck (Houston, TX, USA). Bortezomib (PS-341), another selective proteasome inhibitor and approved by FDA for the treatment of multiple myeloma, was also obtained from Selleck (Houston, TX, USA). Tissue protein extraction buffer was supplied from Wolsen (Beijing, China). Polyclonal antibodies against PTEN, total-Akt, p-Akt and glyceraldehyde-3-phosphate dehydrogenase (GAPDH) and monoclonal antibody against Ubiquitin were from Cell Signaling Technology (Danvers, MA, USA); NEDD4 polyclonal antibody was from Proteintech (Chicago, 
IL, USA). Horseradish peroxidase-conjugated goat anti-rabbit IgG or goat anti-mouse IgG was used as the secondary antibody (Sigma-Aldrich). All other chemicals and materials were bought from local commercial sources.

\section{Animals}

Seventy five male Sprague-Dawley (SD) rats weighing from 180 to $220 \mathrm{~g}$ were used in this study. All animal care and experiments were performed in accordance with the Guide for the Care and Use of Laboratory Animals of Xi'an Jiaotong University Animal Experiment Centre. All protocols used in this study were approved by the Laboratory Animal Care Committee of Xi'an Jiaotong University. The rats were randomly divided into five groups: control group (Con group, $n=15$ ), MCT treatment group (MCT group, $\mathrm{n}=15$ ), MCT plus vehicle (Dimethylsulfoxide, DMSO) treatment group (MCT + vehicle group, $\mathrm{n}=15)$, MCT plus MG-132 treatment group (MCT + MG-132 group, $\mathrm{n}=15$ ) and MCT plus bortezomib treatment group (MCT + bortezomib group, $\mathrm{n}=15$ ). All of the groups were kept in the same room with a stable ambient temperature of $18^{\circ} \mathrm{C}-22^{\circ} \mathrm{C}$ and housed in wire cages with free access to a standard diet and tap water, and were subjected to the same light/dark cycle.

\section{Generation of PAH models and drug treatment}

MCT was dissolved in $1 \mathrm{~mol} / \mathrm{L} \mathrm{HCl}$, and then titrated with $1 \mathrm{~mol} / \mathrm{L} \mathrm{NaOH}$ to $\mathrm{pH} 7.4$ with the final concentrations of $20 \mathrm{mg} / \mathrm{mL}$. Both MG-132 and bortezomib were dissolved in DMSO, and then diluted with PBS to a final concentration of $0.1 \mathrm{mg} / \mathrm{mL}$, while the final concentration of DMSO was $0.5 \%$. DMSO was diluted with PBS to a final concentration of $0.5 \%$ as a vehicle for MG-132 and bortezomib. The PAH model was established by a single intraperitoneal injection of $60 \mathrm{mg} / \mathrm{kg}$ of body weight MCT on day 1. DMSO, MG-132 and bortezomib (both $0.1 \mathrm{mg} / \mathrm{kg}$ of body weight) were administered by daily intraperitoneal injection for 4 weeks until the rats were sacrificed. Rats in the control group received an equal volume of vehicle solution (1 $\mathrm{mol} / \mathrm{L} \mathrm{HCl}$ titrated with $1 \mathrm{~mol} / \mathrm{L} \mathrm{NaOH}$ to $\mathrm{pH} 7.4$ ) on day 1 , and then were administered with vehicle (DMSO diluted with PBS) by daily intraperitoneal injection for 4 weeks. Four of the fifteen rats died in the MCT group, three of the fifteen rats 
died in the MCT + vehicle group, whereas one of the fifteen rats died in the MCT + MG-132 group twenty-eight days after MCT injection. Hemodynamic measurement was performed and tissue samples were collected for morphometric and Western blot analysis when rats were sacrificed 28 days after the MCT injection. Hemodynamic measurement was repeated three times to get an average value for each animal. Western blot was repeated four times using different rat samples.

\section{Measurement of the RVSP and the RVH}

At the end of the experiments, all survived rats were anesthetized with intraperitoneal injections of $10 \%$ chloral hydrate $(0.3 \mathrm{~mL} / \mathrm{kg}$ of body weight $)$ and put in the supine position. A polyvinyl catheter filled with heparin-saline solution was inserted from the right external jugular vein into the right ventricle through the right atrium and tricuspid valve as previously described $[34,35]$. RVSP was assumed to be equal to the pulmonary arterial pressure (PAP) in the presence of a normal pulmonary valve. RVSP was monitored using a Grass polygraph (PowerLab; AD Instruments, Sydney, Australia). The analog signals of pressure were digitized with a sampling frequency of $1,000 \mathrm{~Hz}$ and expressed in millimeters of mercury. After hemodynamic measurements, the thorax was opened and the heart was dissected and weighed for calculation of the right ventricular hypertrophy index (RVHI), assessed by the ratio of right ventricular free wall weight divided the sum of the septum plus left ventricular free wall weight $(\mathrm{RV} / \mathrm{LV}+\mathrm{S})$.

\section{Histological and morphometric analysis}

Marginal left lower pulmonary lobes were harvested and immersed in $4 \%$ buffered paraformaldehyde at room temperature overnight, and then embedded in paraffin wax. Paraffin-embedded tissue was cut at a thickness of $5 \mu \mathrm{m}$ and mounted on glass slides. Tissue sections were stained with hematoxylin and eosin (HE) as previously described $[34,36]$. Pulmonary vascular remodeling was assessed by measuring the percentage medial wall thickness (\%MT) of vessels (diameter, 50-200 $\mu \mathrm{m})$. Vessel external diameter (ED) and medial wall thickness (MT) were measured using a light microscope by an observer who was blinded to the treatments of the rats. 
Distorted arteries were not used for the measurement. The \% MT was calculated as (2MT/ED) x 100.

\section{Western blot analysis}

After rats were sacrificed, peripheral lung tissues were dissected and frozen at $-80{ }^{\circ} \mathrm{C}$. Tissues were homogenized in RIPA protein extraction buffer containing protease inhibitors, phosphatase inhibitors and PMSF. Tissue lysates were centrifuged at $10,000 \mathrm{rpm}$ at $4^{\circ} \mathrm{C}$ for $20 \mathrm{~min}$ and the supernatant was collected as total protein. The protein concentrations were measured using BCA protein assay kit (Pierce, Rockford, IL, USA). The lung tissue lysates were separated by $10 \%$ sodium dodecyl sulfate-polyacrylamide gel electrophoresis. Then, the proteins were transferred onto nitrocellulose membrane in a Bio-Rad Trans-Blot system. After being blocked with $5 \%$ powered non-fat dry milk, membranes were incubated with the following primary antibodies (1:1000 dilution): PTEN, total-Akt, p-Akt , NEDD4, ubiquitin and glyceraldehyde-3-phosphate dehydrogenase (GAPDH) on a rocking platform at $4{ }^{\circ} \mathrm{C}$ overnight, and were then washed and incubated with 1:10,000 diluted horseradish peroxidase-conjugated secondary antibody at room temperature for $1 \mathrm{~h}$. After extensive washing with PBST solution, protein-antibody complexes were visualized using the enhanced chemiluminescence detection system (Amersham Bioscience). The optical density of the bands was measured using Quality One software (Bio-Rad).

\section{Statistical analysis}

Values were presented as mean \pm S.D. Data were analyzed using one-way ANOVA followed by a Tukey post hoc test. $\mathrm{P}<0.05$ was considered to represent significant differences between groups.

\section{Results}


To determine the effects of proteasome inhibitors MG-132 and bortezomib treatments on MCT-induced PAH in rats, RVSP and RVHI were examined. As shown in Fig. 1A, RVSP measured at day 28 in rats treated with MCT $(49.36 \pm 3.41 \mathrm{mmHg})$ was significantly elevated compared with control rats $(27.76 \pm 2.69 \mathrm{mmHg} ; \mathrm{P}<0.05)$. However, treatment of PAH rats with proteasome inhibitor MG-132 for 4 weeks dramatically reduced the RVSP $(37.17 \pm 4.92 \mathrm{mmHg})$ compared with the vehicle-treated PAH rats $(50.26 \pm 3.78 \mathrm{mmHg} ; \mathrm{P}<0.05)$, bortezomib also reduced RVSP in PAH rats $(35.88 \pm 4.31 \mathrm{mmHg} ; \mathrm{P}<0.05$ vs. MCT + vehicle-treated group). Similar results were also observed in the measurement of the index of RVH (Fig. 1B), another parameter for PAH. The RVHI in MCT-treated rats was $0.63 \pm 0.06$, which was significantly higher than that in the control rats $(0.27 \pm 0.03 ; \mathrm{P}<0.05)$. After administration of MG-132 or bortezomib in MCT-treated rats (MCT + MG-132 group and MCT + bortezomib group), RVHI dramatically declined to $0.48 \pm 0.07$ and $0.46 \pm 0.02$, respectively (MCT + MG-132 group and MCT + bortezomib group vs. $\mathrm{MCT}+$ vehicle group; both $\mathrm{P}<0.05)$. These results suggest that inhibition of proteasome effectively suppressed the development of PAH.

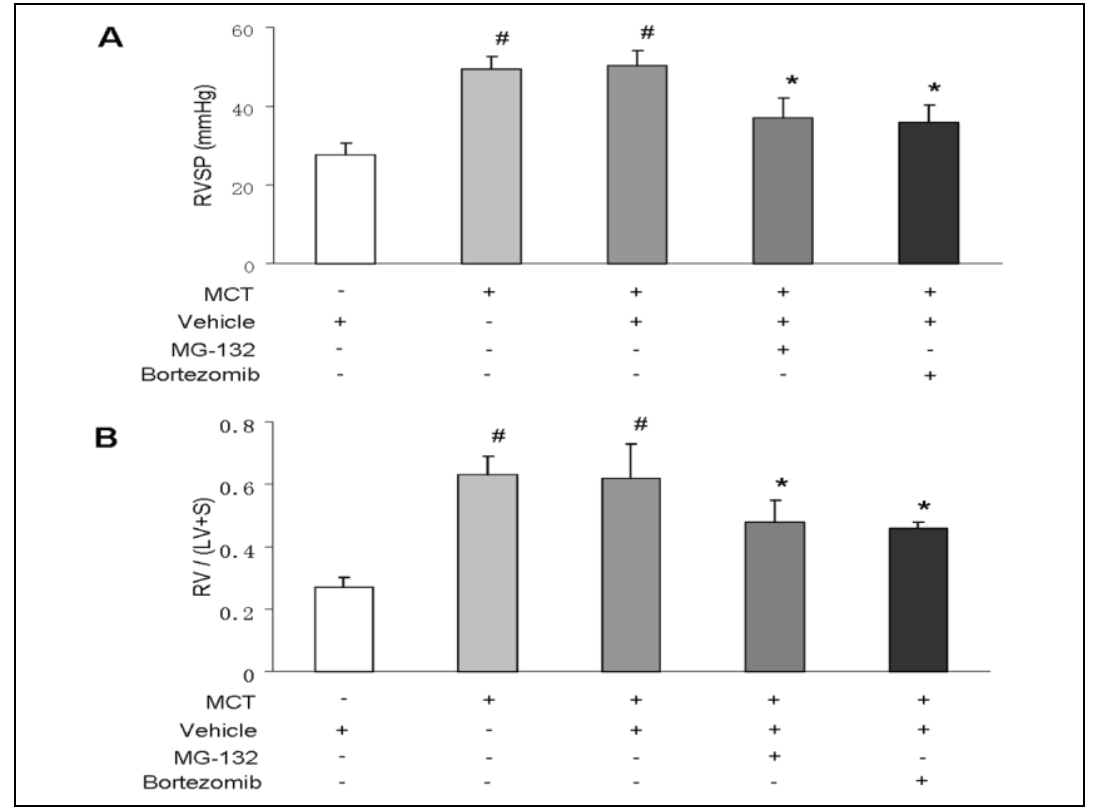

Figure 1: Proteasome inhibition prevents MCT-induced pulmonary arterial hypertension. (A) Changes of RVSP in each group. The RVSP was measured by cannulation of the right jugular vein $(n=11-15)$. (B) Changes of RVHI in each group $(\mathrm{n}=11-15) .{ }^{\#} \mathrm{P}<0.05$ versus control group; $* \mathrm{P}<0.05$ versus $\mathrm{MCT}+$ vehicle group. RVSP: right ventricle systolic pressure; RVHI: right ventricular hypertrophy index. 


\section{Effect of proteasome inhibition on MCT-induced pulmonary arterial remodeling}

Pulmonary arterial remodeling plays an important role in pathogenesis of PAH. We next examined the effect of proteasome inhibition on MCT-induced remodeling of the pulmonary artery using histological analysis. Fig. 2A shows the HE-staining results of the marginal left lower pulmonary lobes. The MCT-treated rats showed a significant increase in medial wall thickness compared with control rats at week 4; this was accompanied by an increase of PASMCs number in the medial layer of pulmonary artery. Proteasome inhibitors MG-132 and bortezomib significantly prevented MCT-induced increase of medial wall thickness and PASMCs number. Furthermore, the quantitative morphometric analysis (Fig. 2B) confirmed that proteasome inhibitors MG-132 and bortezomib attenuated pulmonary arterial remodeling. \%MT in MCT-induced model of PAH was significantly increased [MCT group $(52.13 \pm 7.40) \%$ vs. control group $(28.41 \pm 5.02) \%$; P < 0.05$]$, while the presence of either MG-132 or bortezomib reduced \%MT in PAH model [MCT + MG-132 group $(39.03 \pm 8.01) \%$ and MCT + bortezomib group (37.82 \pm 3.67$) \%$ vs. MCT + vehicle group (52.07 \pm 9.62$) \%$; both $\mathrm{P}<0.05]$. These results indicate that proteasome inhibition dramatically inhibited the pulmonary arterial remodeling by suppressing the proliferation of PASMCs.

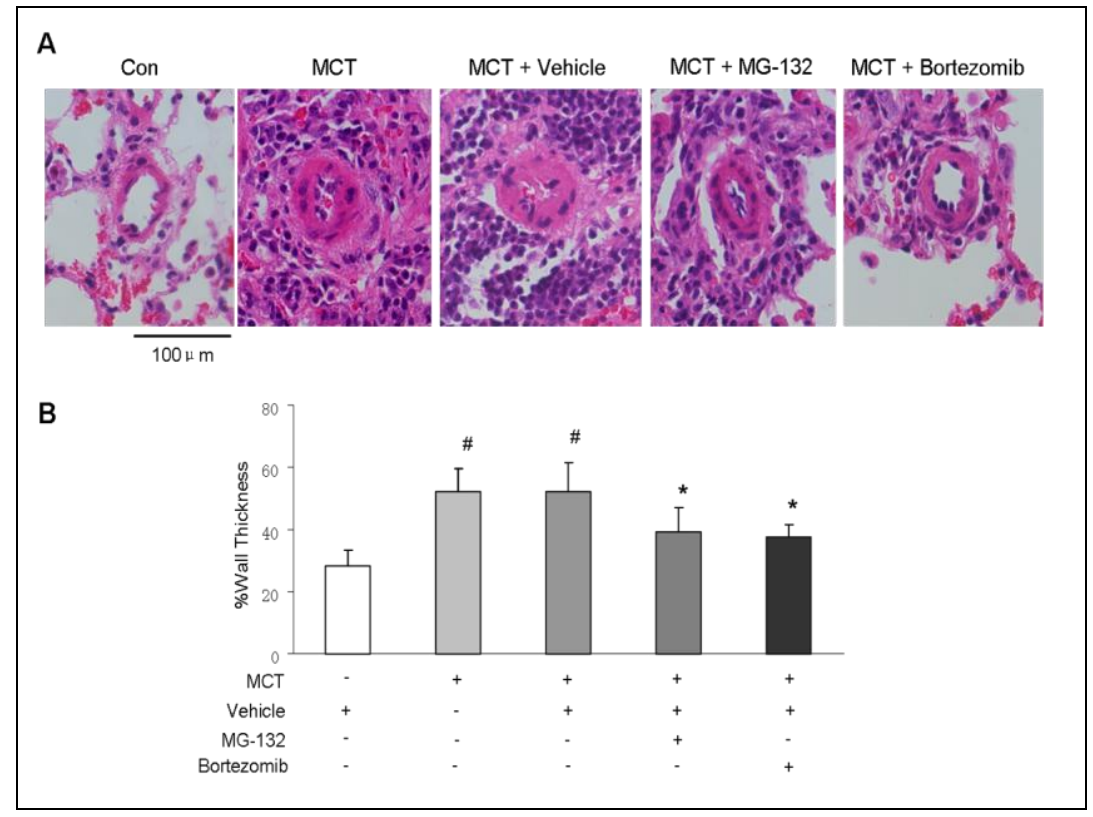


Figure 2: Proteasome inhibition prevents MCT-induced pulmonary arterial remodeling. (A) Representative HE-staining photomicrographs of small pulmonary vessels in different groups (magnification $\times 400$ ). (B) Quantitative analysis of the wall thickness of pulmonary artery $(\mathrm{n}=11-15) .{ }^{*} \mathrm{P}<0.05$ versus control group; $* \mathrm{P}<0.05$ versus MCT + vehicle group. Con: control group; MCT: Monocrotaline-treated group; MCT + MG-132: monocrotaline and MG-132-treated group; MCT + bortezomib: monocrotaline and bortezomib-treated group.

\section{Proteasome inhibition prevents MCT-induced pulmonary arterial remodeling by increasing PTEN expression and inhibiting PI3K/Akt pathway}

It has been shown that dysregulation of PTEN-PI3K/Akt signaling axis is involved in MCT-induced rats PAH model, and down-regulation of PTEN is associated with PASMCs proliferation in PAH [14, 37]. In addition, previous studies have suggested that PTEN is degraded by UPS, which then leads to the development of numerous types of human cancer by promoting tumor cell proliferation [38]. PAH is a non-oncologic and proliferative disease majorly caused by PASMCs proliferation/migration and has similar pathological mechanisms with cancer. Therefore, it is important to determine whether UPS also promotes the degradation of PTEN in MCT-induced model of PAH. As shown in Figure 3A, MCT significantly reduced the protein level of PTEN to $0.48 \pm 0.004$-fold compared with control group $(\mathrm{P}<0.05)$, while treatment of PAH rats with either MG-132 or bortezomib restored the PTEN protein levels to $1.00 \pm 0.189$-fold and $1.23 \pm 0.194$-fold over control, respectively [both $\mathrm{P}<0.05$ vs. MCT + vehicle group $(0.61 \pm 0.049$-fold $)$ ], suggesting that inhibition of proteasome function suppresses UPS-mediated degradation of PTEN.

Loss of PTEN enhances PI3K/Akt signaling activity, which is associated with a variety of types of cell proliferation. We therefore examined the phosphorylation of Akt in lung tissues of PAH models. Fig. 3B shows that the phosphorylation level of Akt was increased to $2.37 \pm 0.194$-fold over control in MCT-induced PAH model, while treatment PAH rats with proteasome inhibitors MG-132 or bortezomib reduced the levels of Akt phosphorylation to $1.77 \pm 0.044$-fold and $1.81 \pm 0.156$-fold over 
control, respectively [MCT + MG-132 group and MCT + bortezomib group vs. MCT + vehicle group $(2.20 \pm 0.005$-fold $)$, both $\mathrm{P}<0.05]$, indicating that inhibition of proteasome function suppresses the degradation of PTEN and subsequently increases PI3K/Akt pathway.

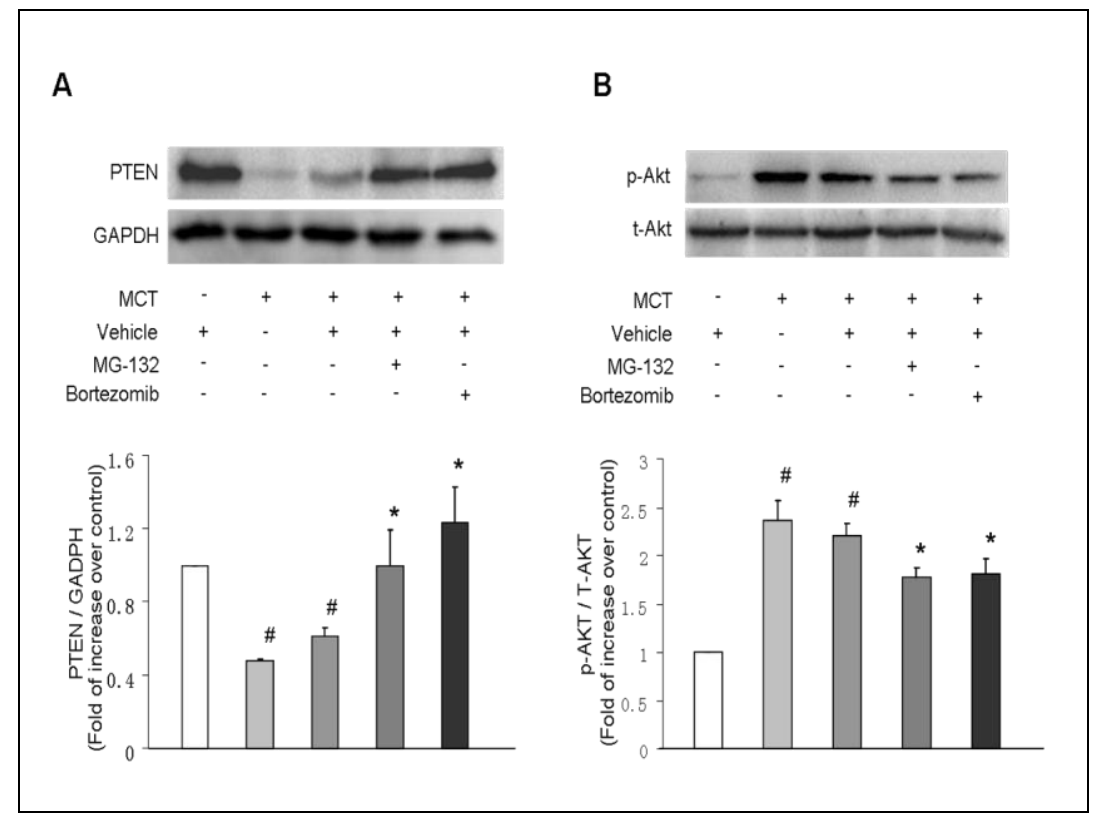

Figure 3: Proteasome inhibition raises PTEN protein level and inhibits PI3K/Akt signaling in MCT-induced PAH rats. (A) Representative western blot of PTEN protein level in lung tissues from different groups and its quantification analysis. GAPDH served as a loading control ( $n=4$ per group). (B) Akt phosphorylation was analyzed by Western blot in lung tissues from different groups. A representative blot and quantification of bands are shown ( $\mathrm{n}=4$ per group). ${ }^{\#} \mathrm{P}<0.05$ versus control group; * $\mathrm{P}<0.05$ versus MCT + vehicle group.

\section{E3 ligase NEDD4 is elevated in MCT-induced PAH rats}

It has been shown that HECT-domain protein NEDD4, an ubiquitin E3 ligase in UPS, negatively regulates PTEN stability by catalyzing PTEN ubiquitinated degradation in human cancer cells [38]. The above results indicated that UPS promoted degradation of PTEN in MCT-induced model of PAH rats. We therefore examined the protein level of NEDD4 in lung lysates here. Figure 4 indicates that MCT dramatically increased NEDD4 protein level, which raised to $1.57 \pm 0.071$-fold compared with control rats $(\mathrm{P}<0.05)$; while treatment of $\mathrm{PAH}$ rats with MG-132 or bortezomib did 
not change the protein level of NEDD4 [1.65 \pm 0.079 -fold and $1.59 \pm 0.033$-fold over control, respectively; both $\mathrm{P}>0.05$ vs. MCT + vehicle group $(1.79 \pm 0.224$-fold $)]$. These results suggest that E3 ligase NEDD4 might be involved in UPS-mediated PTEN degradation in MCT-induced model of PAH, whereas inhibition proteasome function does not affect MCT-induced the elevation of NEDD4.

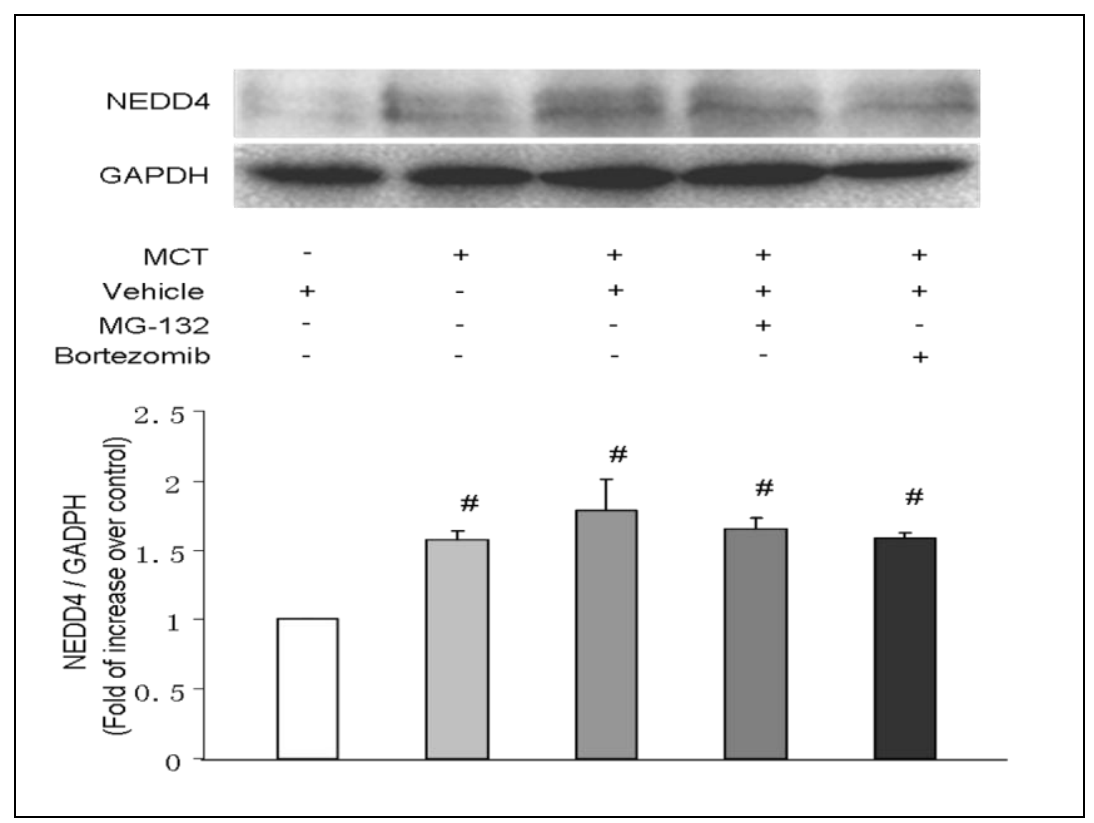

Figure 4: NEDD4 protein level is elevated in MCT-induced PAH rats. The expression of NEDD4 was analyzed using Western blot in lung tissues from different groups. GAPDH served as a loading control. A representative blot and quantification of bands are shown ( $\mathrm{n}=4$ per group). ${ }^{\#} \mathrm{P}<0.05$ versus control group.

\section{Proteasome inhibition elevates ubiquitinated-PTEN protein in MCT-induced PAH rats}

The above results indicated that reduction of PTEN might be associated with NEDD4-related and UPS-mediated protein degradation in MCT-induced model of PAH. To further investigate the degradation of ubiquitinated-PTEN protein by proteasome, ubiquitinated-PTEN protein was detected by immunobloting with an anti-ubiquitin antibody in rats. As shown in Fig. 5, treatment of PAH rats with either MG-132 or bortezomib increased the level of ubiquitinated-PTEN protein compared to control rats or MCT-induced PAH rats [1.98 \pm 0.092 -fold and $2.02 \pm 0.051$-fold 
over control, respectively; both $\mathrm{P}<0.05$ vs. Con group or MCT + vehicle group $(0.98$ \pm 0.019 -fold)], leading to the marked accumulation of ubiquitinated-PTEN protein. These results confirm that proteasome inhibition appears to inhibit proteasome-dependent degradation of ubiquitinated PTEN protein in PAH rats.

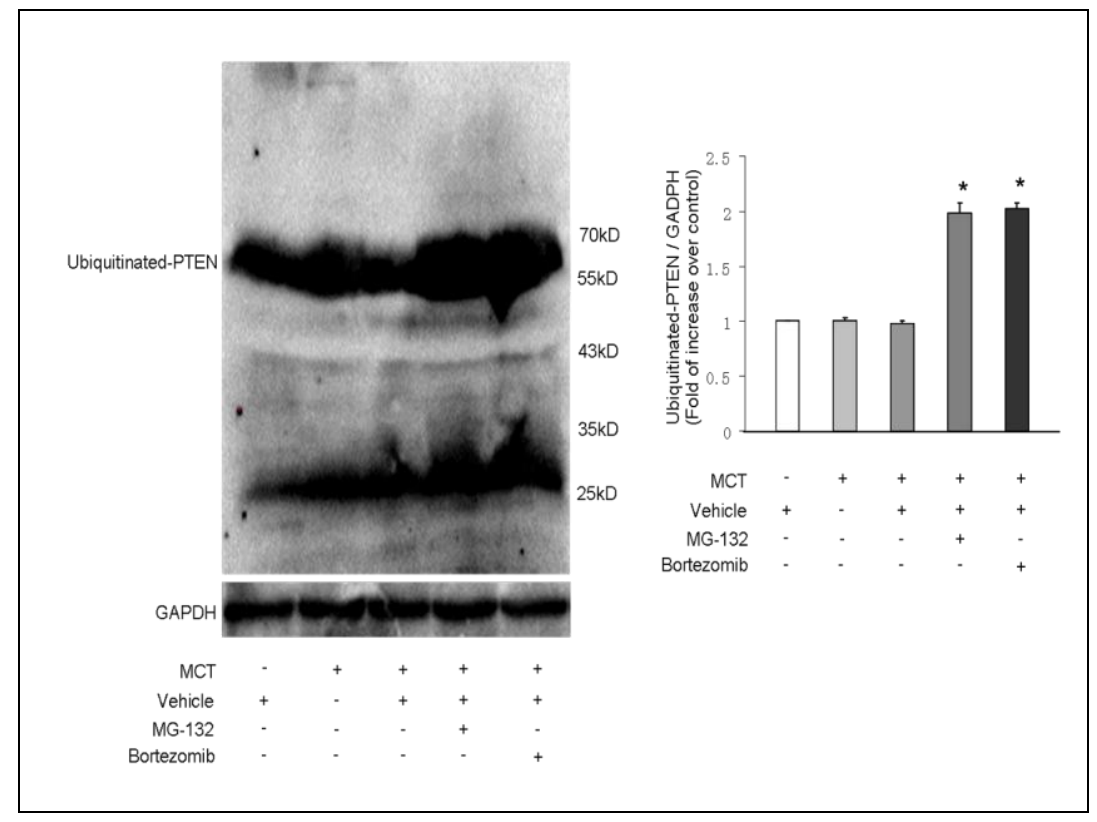

Figure 5: Proteasome inhibition elevates the level of ubiquitinated-PTEN protein in MCT-induced model of PAH. Representative western blot of ubiquitinated-PTEN protein and quantification of bands are shown in lung tissues from different groups. GAPDH served as a loading control ( $\mathrm{n}=4$ per group). $* \mathrm{P}<0.05$ versus MCT + vehicle group.

\section{Discussion}

In this study, we have demonstrated that the inhibition of proteasome function by MG-132 or bortezomib significantly suppresses pulmonary vascular remodeling and the development of MCT-induced PAH in rats. This effect is associated with a suppression of UPS-mediated PTEN degradation and subsequent inhibition of PI3K/Akt pathway, and finally an inhibition of PASMCs proliferation. In addition, the up-regulation of E3 ligase NEDD4 might be associated with the degradation of PTEN. Our study provides a novel molecular basis whereby inhibition of proteasome 
function might have a potential value in the management of PAH via amelioration of vascular remodeling.

PTEN is a major tumor suppressor that antagonizes endogenous PI3K/Akt signaling by converting PIP3 to phosphatidyl inositol-4, 5-trisphosphate (PIP2). It has been shown that PI3K/Akt signaling is involved in human PASMCs proliferation leading to vascular remodeling [39]. Frequent genetic aberrations of PTEN are related to various sporadic human cancers, including glioblastoma, melanoma, endometrial, lung, breast and prostate cancers [40-42]. Furthermore, recent discoveries of post-translational regulation of PTEN by phosphorylation, oxidation, acetylation and ubiquitination/deubiquitination have generated widespread speculation on the implications of PTEN protein function/stability in tumorigenic processes [5, 15, 41]. Several studies have also suggested that PTEN effectively suppresses cardiomyocyte hypertrophy and PASMCs proliferation [37, 43, 44]. In this study, we found that the protein level of PTEN was significantly reduced in MCT-induced PAH model, along with the increase of Akt phosphorylation, as well as PASMCs proliferation and pulmonary vascular remodeling, suggesting that reduction of PTEN expression induces PASMCs proliferation and pulmonary vascular remodeling via activation of PI3K/Akt signaling pathway in MCT-induced PAH model.

UPS recognizes and degrades damaged, altered, misfolded or unnecessary proteins in order to maintain cell functionality and viability [23]. E3 ligase determines substrate specificity by recognizing lysine residues on the targeted protein. The deregulation of UPS components has been observed, especially up-regulation of E3 ubiquitin ligases, in numerous types of cancer, including lung cancer; and their deregulation has been shown to contribute to cancer development by promoting cell proliferation [45-48]. Previous studies have shown that elevated function of UPS suppresses PTEN protein expression and aberrant up-regulation of ubiquitin ligase NEDD4 specifically recognizes and results in PTEN degradation in several cancers and neurological diseases. Further studies have demonstrated that the strategy of increasing PTEN protein level by suppressing UPS-mediated its degradation can inhibit PI3K/Akt signaling pathway-mediated proliferation of tumor cells [26, 28, 29]. The present 
study showed that the protein level of E3 ligase NEDD4 was dramatically elevated in MCT-induced PAH rats. This was accompanied by a reduction of PTEN protein and an increase of Akt phosphorylation, suggesting that the up-regulation of ubiquitin ligase NEDD4 might be associated with PTEN degradation and consequent activation of PI3K/Akt signaling pathway in MCT-induced PAH rats.

Proteasome inhibitor has been approved by US Food and Drug Administration for the treatment of several hematological malignancies in clinic [30]. Such class drug is reported to have potentially protective effects via multiple mechanisms on cardiovascular diseases, neurological disorders and inflammatory diseases [27-29, 49]. Recent study has suggested that inhibition of proteasome function also suppresses PASMCs proliferation in vitro by increasing the expression of negative cell cycle regulatory protein $\mathrm{P} 21^{\mathrm{WAF} 1}$ [32]. In addition, It has been shown that proteasome inhibitors reduce the media and intima thickness of remodeled pulmonary vascular walls by promotion of apoptotic and autophagic death of vascular cells by regulating annexin A1/GATA4 pathway in vivo [33]. The results of the present study further indicated that inhibition of proteasome function by MG-132 or bortezomib prevented the development of MCT-induced PAH in rats by suppressing UPS-mediated PTEN degradation and subsequent inhibition of PI3K/Akt signaling cascade. All these studies including our own suggest that the inhibition of proteasome function might benefit for PAH by protecting several important proteins from degradation. In this study, DMSO used as a vehicle for MG-132 and bortezomib might impact mammalian cells proliferation in vivo. However, since we used the same concentration and volume of DMSO in both control and vehicle-treated MCT-induced PAH rats, the beneficial changes observed in the current study may reflect the effects of proteasome inhibition on the development of PAH.

\section{Conclusion}

Proteasome inhibitors MG-132 and bortezomib suppress the development of MCT-induced PAH in rats by ameliorating vascular remodeling. One of the underlying mechanisms is associated with the inhibition of UPS-mediated PTEN 
degradation and subsequently inhibits PI3K/Akt pathway, and ultimately results in the inhibition of PASMCs proliferation. NEDD4, as an E3 ligase, might be involved in UPS-mediated PTEN degradation. These results indicate that inhibition of ubiquitin proteasome function might be a novel strategy for PAH prevention and treatment.

\section{Conflict of interest statement}

The authors declare that there are no conflicts of interest.

\section{Acknowledgments}

This work was supported by National Science Foundation of China (No.81330002).

\section{References}

[1] Miura Y, Fukumoto Y, Sugimura K, Oikawa M, Nakano M, Tatebe S, et al. Identification of new prognostic factors of pulmonary hypertension. Circ J 2010; 74: 1965-71.

[2] Hanze J, Weissmann N, Grimminger F, Seeger W, Rose F. Cellular and molecular mechanisms of hypoxia-inducible factor driven vascular remodeling. Thromb Haemost 2007; 97: 774-87.

[3] Stenmark KR, Fagan KA, Frid MG. Hypoxia-induced pulmonary vascular remodeling: cellular and molecular mechanisms. Circ Res 2006; 99: 675-91.

[4] Hopkins BD, Hodakoski C, Barrows D, Mense SM, Parsons RE. PTEN function: the long and the short of it. Trends Biochem Sci 2014; 39: 183-90.

[5] Song MS, Salmena L, Pandolfi PP. The functions and regulation of the PTEN tumour suppressor. Nat Rev Mol Cell Biol 2012; 13: 283-96.

[6] Raftopoulou M, Etienne-Manneville S, Self A, Nicholls S, Hall A. Regulation of cell migration by the C2 domain of the tumor suppressor PTEN. Science 2004; 303: 1179-81.

[7] Maehama T, Taylor GS, Dixon JE. PTEN and myotubularin: novel phosphoinositide phosphatases. Annu Rev Biochem 2001; 70: 247-79. 
[8] Cully M, You H, Levine AJ, Mak TW. Beyond PTEN mutations: the PI3K pathway as an integrator of multiple inputs during tumorigenesis. Nature reviews Cancer 2006; 6: 184-92.

[9] Li J, Yen C, Liaw D, Podsypanina K, Bose S, Wang SI, et al. PTEN, a putative protein tyrosine phosphatase gene mutated in human brain, breast, and prostate cancer. Science 1997; 275: 1943-7.

[10] Sansal I, Sellers WR. The biology and clinical relevance of the PTEN tumor suppressor pathway. J Clin Oncol 2004; 22: 2954-63.

[11] Kitagishi Y, Matsuda S. Redox regulation of tumor suppressor PTEN in cancer and aging. Int J Mol Med 2013; 31: 511-5.

[12] Li J, Tanhehco EJ, Russell B. Actin dynamics is rapidly regulated by the PTEN and PIP2 signaling pathways leading to myocyte hypertrophy. American journal of physiology Heart and circulatory physiology 2014; 307: H1618-25.

[13] Xue R, Lei S, Xia ZY, Wu Y, Meng Q, Zhan L, et al. Selective inhibition of PTEN preserves ischaemic post-conditioning cardioprotection in STZ-induced Type 1 diabetic rats: role of the PI3K/Akt and JAK2/STAT3 pathways. Clin Sci (Lond) 2016; 130: 377-92.

[14] Xie X, Wang G, Zhang D, Zhang Y, Zhu Y, Li F, et al. Activation of peroxisome proliferator-activated receptor gamma ameliorates monocrotaline-induced pulmonary arterial hypertension in rats. Biomed Rep 2015; 3: 537-42.

[15] Wang X, Jiang X. Post-translational regulation of PTEN. Oncogene 2008; 27: 5454-63.

[16] Wu CW, Bell RA, Storey KB. Post-translational regulation of PTEN catalytic function and protein stability in the hibernating 13-lined ground squirrel. Biochim Biophys Acta 2015.

[17] Fata JE, Debnath S, Jenkins EC, Jr., Fournier MV. Nongenomic Mechanisms of PTEN Regulation. Int J Cell Biol 2012; 2012: 379685.

[18] Trotman LC, Wang X, Alimonti A, Chen Z, Teruya-Feldstein J, Yang H, et al. Ubiquitination regulates PTEN nuclear import and tumor suppression. Cell 2007; 128: 141-56.

[19] Kriegenburg F, Ellgaard L, Hartmann-Petersen R. Molecular chaperones in targeting misfolded proteins for ubiquitin-dependent degradation. FEBS J 2012; 279: 532-42.

[20] Yi JJ, Ehlers MD. Emerging roles for ubiquitin and protein degradation in neuronal function. Pharmacol Rev 2007; 59: 14-39.

[21] Ravid T, Hochstrasser M. Diversity of degradation signals in the ubiquitin-proteasome system. Nat Rev Mol Cell Biol 2008; 9: 679-90.

[22] Wang X, Robbins J. Heart failure and protein quality control. Circ res 2006; 99: 1315-28.

[23] Willis MS, Townley-Tilson WH, Kang EY, Homeister JW, Patterson C. Sent to destroy: the ubiquitin proteasome system regulates cell signaling and protein quality control in cardiovascular development and disease. Circ res 2010; 106: 463-78.

[24] Rogers N, Paine S, Bedford L, Layfield R. Review: the ubiquitin-proteasome system: contributions to cell death or survival in neurodegeneration. Neuropathol Appl Neurobiol 2010; 36: 113-24.

[25] Schwartz AL, Ciechanover A. Targeting proteins for destruction by the ubiquitin system: implications for human pathobiology. Annu Rev Pharmacol Toxicol 2009; 49: 73-96. 
[26] Wang X, Trotman LC, Koppie T, Alimonti A, Chen Z, Gao Z, et al. NEDD4-1 is a proto-oncogenic ubiquitin ligase for PTEN. Cell 2007; 128: 129-39.

[27] Adams B, Mapanga RF, Essop MF. Partial inhibition of the ubiquitin-proteasome system ameliorates cardiac dysfunction following ischemia-reperfusion in the presence of high glucose. Cardiovasc Diabetol 2015; 14: 94.

[28] Drinjakovic J, Jung H, Campbell DS, Strochlic L, Dwivedy A, Holt CE. E3 ligase Nedd4 promotes axon branching by downregulating PTEN. Neuron 2010; 65: 341-57.

[29] Christie KJ, Martinez JA, Zochodne DW. Disruption of E3 ligase NEDD4 in peripheral neurons interrupts axon outgrowth: Linkage to PTEN. Mol Cell Neurosci 2012; 50: 179-92.

[30] Ruggeri B, Miknyoczki S, Dorsey B, Hui AM. The development and pharmacology of proteasome inhibitors for the management and treatment of cancer. Adv Pharmacol 2009; 57: 91-135.

[31] Moore BS, Eustaquio AS, McGlinchey RP. Advances in and applications of proteasome inhibitors. Curr Opin Chem 2008; 12: 434-40.

[32] Li M, Dong X, Liu Y, Sun X, Li Z, He J. Inhibition of ubiquitin proteasome function suppresses proliferation of pulmonary artery smooth muscle cells. Naunyn Schmiedebergs Arch Pharmacol 2011; 384: 517-23.

[33] Ibrahim YF, Wong CM, Pavlickova L, Liu L, Trasar L, Bansal G, et al. Mechanism of the susceptibility of remodeled pulmonary vessels to drug-induced cell killing. J Am Heart Assoc 2014; 3 : e000520.

[34] Kazama K, Okada M, Yamawaki H. A novel adipocytokine, omentin, inhibits monocrotaline-induced pulmonary arterial hypertension in rats. Biochem Bioph Res Co 2014; 452: 142-6.

[35] Okada M, Harada T, Kikuzuki R, Yamawaki H, Hara Y. Effects of Telmisartan on Right Ventricular Remodeling Induced by Monocrotaline in Rats. J Pharmacol Sci 2009; 111: 193-200. [36] Usui T, Sakatsume T, Nijima R, Otani K, Kazama K, Morita T, et al. Death-associated protein kinase 3 mediates vascular structural remodelling via stimulating smooth muscle cell proliferation and migration. Clin Sci 2014; 127: 539-48.

[37] Ravi Y, Selvendiran K, Meduru S, Citro L, Naidu S, Khan M, et al. Dysregulation of PTEN in Cardiopulmonary Vascular Remodeling Induced by Pulmonary Hypertension. Cell Biochemistry and Biophysics 2011; 67: 363-72.

[38] Wang XJ, Trotman LC, Koppie T, Alimonti A, Chen ZB, Gao ZH, et al. NEDD4-1 is a proto-oncogenic ubiquitin ligase for PTEN. Cell 2007; 128: 129-39.

[39] Goncharova EA, Ammit AJ, Irani C, Carroll RG, Eszterhas AJ, Panettieri RA, et al. PI3K is required for proliferation and migration of human pulmonary vascular smooth muscle cells. Am $\mathrm{J}$ Physiol-Lung C 2002; 283: L354-L63.

[40] Shi YJ, Paluch BE, Wang XJ, Jiang XJ. PTEN at a glance. J Cell Sci 2012; 125: 4687-92.

[41] Chalhoub N, Baker SJ. PTEN and the PI3-kinase pathway in cancer. Annu Rev Pathol 2009; 4: 127-50.

[42] Hollander MC, Blumenthal GM, Dennis PA. PTEN loss in the continuum of common cancers, rare syndromes and mouse models. Nat Rev Cancer 2011; 11: 289-301. 
[43] Oudit GY, Penninger JM. Cardiac regulation by phosphoinositide 3-kinases and PTEN.

Cardiovasc Res 2009; 82: 250-60.

[44] Oudit GY, Sun H, Kerfant BG, Crackower MA, Penninger JM, Backx PH. The role of phosphoinositide-3 kinase and PTEN in cardiovascular physiology and disease. J Mol Cell Cardiol 2004; 37: 449-71.

[45] Chen H, Wang N, Yang G, Guo Y, Shen Y, Wang X, et al. The expression and function of E3 ligase SIAH2 in acute T lymphoblastic leukemia. Leuk Res 2016; 42: 28-36.

[46] Kitagawa K, Kitagawa M. The SCF-type E3 Ubiquitin Ligases as Cancer Targets. Curr Cancer Drug Targets 2016; 16: 119-29.

[47] Choi HH, Phan L, Chou PC, Su CH, Yeung SC, Chen JS, et al. COP1 enhances ubiquitin-mediated degradation of p27Kip1 to promote cancer cell growth. Oncotarget 2015; 6: 19721-34.

[48] Kalinichenko VV, Moreno P, Lara-Chica M, Soler-Torronteras R, Caro T, Medina M, et al. The Expression of the Ubiquitin Ligase SIAH2 (Seven In Absentia Homolog 2) Is Increased in Human Lung Cancer. Plos One 2015; 10: e0143376.

[49] Chen X, Li SL, Wu T, Liu JD. Proteasome inhibitor ameliorates severe acute pancreatitis and associated lung injury of rats. World J Gastroenterol 2008; 14: 3249-53. 